\title{
Vascular differentiation of multipotent spermatogonial stem cells derived from neonatal mouse testis
}

\author{
Ji-Eun $\mathrm{Im}^{1}$, Sun-Hwa Song ${ }^{2}$, Ji Yeon Kim², \\ Koung Li Kim², Sang Hong Baek ${ }^{3}$, \\ Dong Ryul Lee ${ }^{1}$ and Wonhee Suh ${ }^{2,4}$
}

\author{
${ }^{1}$ Department of Biomedical Science \\ College of Life Science \\ CHA University \\ Pochon 487-010, Korea \\ ${ }^{2}$ College of Pharmacy \\ Ajou University \\ Suwon 443-749, Korea \\ ${ }^{3}$ Cardiovascular Center \\ Seoul St. Mary's Hospital \\ College of Medicine \\ The Catholic University of Korea \\ Seoul 137-701, Korea \\ ${ }^{4}$ Corresponding author: Tel, 82-31-219-3445; \\ Fax, 82-31-219-3675; E-mail, wsuh@ ajou.ac.kr \\ http://dx.doi.org/10.3858/emm.2012.44.4.034
}

Accepted 16 January 2012

Available Online 19 January 2012

Abbreviations: BMP-4, bone morphogenic protein-4; EB, embryonic body; EC, endothelial cell; EPC, endothelial progenitor cell; ESC, embryonic stem cell; mSSC, multipotent spermatogonial stem cell; SMA, smooth muscle actin; VSMC, vascular smooth muscle cell

\begin{abstract}
We previously reported the successful establishment of embryonic stem cell (ESC)-like multipotent spermatogonial stem cells (mSSCs) from neonatal mouse testis. Here, we examined the ability of mSSCs to differentiate into vascular endothelial cells and smooth muscle cells, and compared to that of mouse ESCs. We used real-time reverse transcriptase polymerase chain reaction and immunohistochemistry to examine gene expression profiles of $\mathrm{mSSC}$ and ESCs during in vitro vascular differentiation. Both mSSCs and ESCs exhibited substantial increase in the expression of mesodermal markers, such as Brachyury, Flk1, Mesp1, Nkx2.5, and Islet1, and a decrease in the expression of pluripotency markers, such as Oct3/4 and Nanog dur-
\end{abstract}

ing the early stage of differentiation. The mRNA levels of vascular endothelial (VE)-cadherin and CD31 gradually increased in both differentiated mSSCs and ESCs. VE-cadherin- or CD31-positive cells formed sprouting branch-like structures, as observed during embryonic vascular development. At the same time, vascular smooth muscle cell-specific markers, such as myocardin and $\alpha$-smooth muscle actin (SMA), were also highly expressed in differentiated mSSCs and ESCs. Immunocytochemical analysis revealed that the differentiated cells expressed both $\alpha$-SMA and SM22- $\alpha$ proteins, and exhibited the intracellular fibril structure typical of smooth muscle cells. Overall, our findings showed that mSSCs have similar vascular differentiation abilities to those of ESCs, suggesting that mSSCs may be an alternative source of autologous pluripotent stem cells for vascular regeneration.

Keywords: cell differentiation; endothelial cells; multipotent stem cells; myocytes, smooth muscle; spermatogonia

\section{Introduction}

Constriction or obstruction of blood vessels causes restricted blood flow in nearby tissues, leading to ischemic vascular diseases, such as stroke, myocardial infarction, and peripheral arterial disease. For the treatment of ischemic vascular diseases, several studies have attempted to generate new blood vessel to restore blood circulation and minimize tissue damages.

In 1997, it was first discovered that endothelial progenitor cells (EPCs) circulating in adult peripheral bloods could promote vascular regeneration in ischemic tissues (Asahara et al., 1997). Since then, the therapeutic potential of EPCs has been investigated for the treatment of many ischemic diseases (Rafii and Lyden, 2003; Shantsila et al., 2007; Park et al., 2011). However, the limited number of EPCs in adult tissues and the lack of in vitro expansion methods have made it difficult to obtain sufficient EPCs for clinical application. Moreover, the EPCs harvested from patients with ischemic vascular diseases had a reduced ability to repair 
A

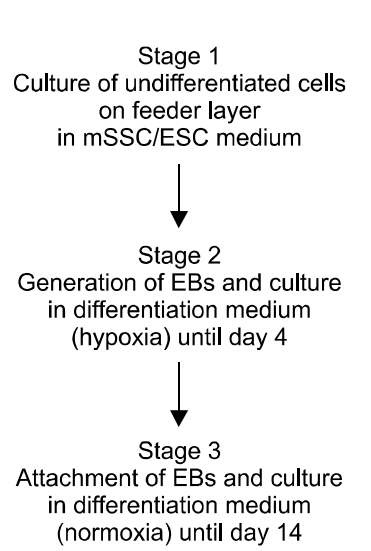

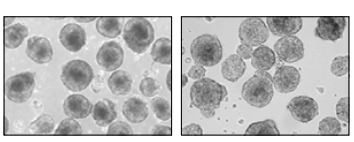

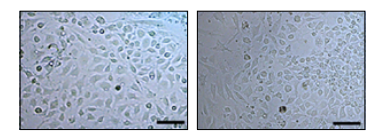

Figure 1. Experimental scheme of in vitro vascular differentiation process and morphological changes of mSSCs and ESCs during differentiation. (A) Experimental scheme showing in vitro differentiation into vascular cells, $(B, C)$ Representative images of mSSCs (B) and ESCs (C) at three different stages. Undifferentiated mSSCs and ESCs were cultured on STO in ESC medium (stage 1). Undifferentiated cells were aggregated into EBs by using the standard hanging drop method. EBs were incubated in vascular differentiation medium containing BMP4, VEGF, activin A, and bFGF for 4 days (stage 2). EBs were then attached into gelatin-coated plates and cultured until day 14 (stage 3). Scale bar is $50 \mu \mathrm{m}$.

vasculature as compared to those from healthy individuals (Hill et al., 2003). As such, autologous EPC transplantation into patients with ischemic vascular diseases has only marginal therapeutic efficacy, as it is the case with transplantation of other adult stem cells such as mesenchymal stem cells and adipose-derived stem cells. Because of these drawbacks, it is important to develop new sources of autologous stem cells that highly self-renewal and are independent of the host's disease status.

Several years ago, autologous multipotent stem cells were established from neonatal and adult mice testes and were named as multipotent spermatogonial stem cells (mSSCs) (Kanatsu-Shinohara et al., 2004; Guan et al., 2006; Seandel et al., 2007). mSSCs exhibited high self-renewal properties and express pluripotency - related genes (Oct3/4, Nanog, SSEA-1, and alkaline phosphatase), similar to those observed in mouse embryonic stem cells (ESCs). Recent reports have demonstrated the successful establishment of mSSCs from human testes, indicating that $\mathrm{mSSC}$ are a new promising source of autologous stem cells that do not have the same problems as adult stem cells (Conrad et al., 2008; Kossack et al., 2009).

Therefore, the present study aimed to examine the ability of mSSCs to differentiate into vascular endothelial cells and smooth muscle cells for the treatment of ischemic vascular diseases. mSSCs used in the present study was previously established

from SSCs isolated from neonatal mouse testis based on the modified Shinohara's culture method, and their ESC-like properties were extensively characterized (Kim et al., 2010).

\section{Results}

\section{Mesodermal differentiation of mSSCs}

The mSSCs used in the present study were previously characterized to have ESC-like properties in cell morphology, expression of pluripotent stem cell markers, and DNA methylation profiles (Kim et al., 2010). To induce vascular differentiation of mSSCs, embryonic bodies (EBs) were generated by the hanging drop method and were incubated in differentiation media containing vascular endothelial growth factor (VEGF), bone morphogenic protein (BMP) 4, activin A, and basic fibroblast growth factor (bFGF), which are critical modulators of early mesodermal differentiation (Pearson et al., 2008). During the differentiation process, EBs were grown into a uniform size in the suspension culture for 4 days and were further differentiated by attachment to gelatin-coated dishes and cultivation until day 14 . Throughout the vascular differentiation process, cells with a variety of morphologies emerged from EB outgrowths (Figure 1).

To measure gene expression changes associated with early mesodermal differentiation, differentiated cells were collected at various time points during in vitro differentiation and mRNA levels of pluripotency marker genes and mesoderm lineage-related genes were analyzed using real-time reverse transcriptase polymerase chain reaction (RT-PCR). In both mSSCs and ESCs the expression of Oct3/4 and Nanog gradually decreased, while the expression of Brachyury, early mesodermal marker, dramatically increased at day 5 after vascular differentiation (Figures 2A and 2B). Other mesodermal markers including Flk1 and Mesp1 were maximally expressed 4 days later (Figure 2B), which was confirmed by fluorescence-activated cell sorter (FACS) and immunocytochemical analyses (Figures $2 \mathrm{C}$ and 2D). On day 9 and 14 of vascular differentiation, significant number of differentiated cells from mSSCs and ESCs were positively stained with anti-Flk 1 lgG.

In addition, cardiac transcription factors involved in early heart development, Islet1 and Nkx2.5, were highly expressed at high levels from days 9 to 14 . However, at day 9, the expression of these genes was weaker in differentiated mSSCs than in differentiated ESCs, suggesting that mSSCs have less potential for cardiac differentiation than ESCs have. 
A

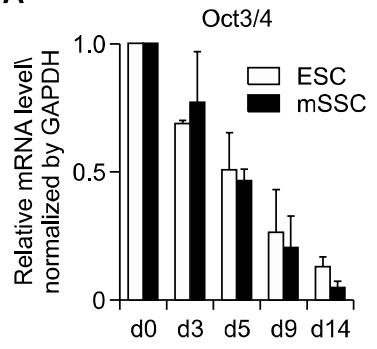

B
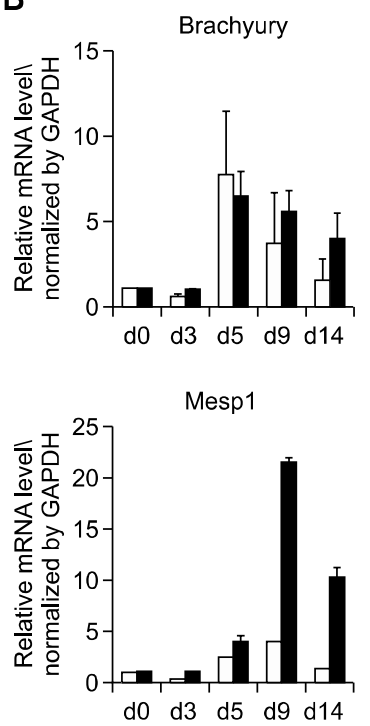

Nkx2.5

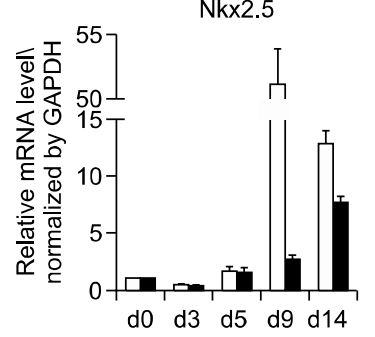

Nanog

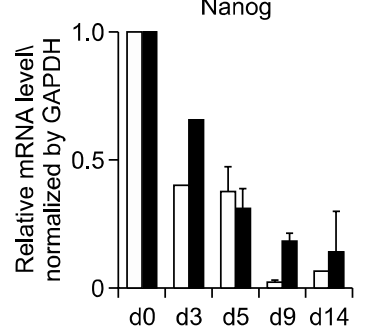

Flk1
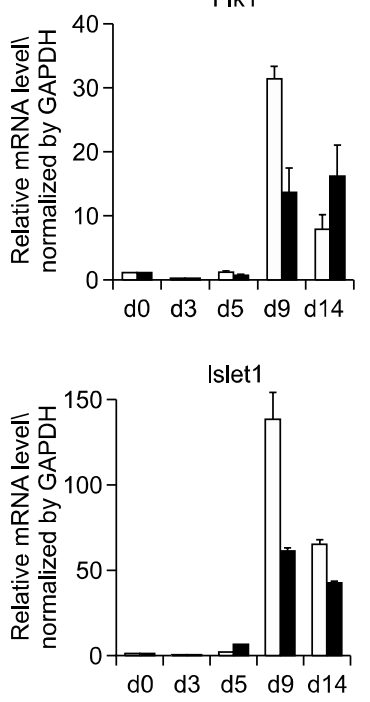

C
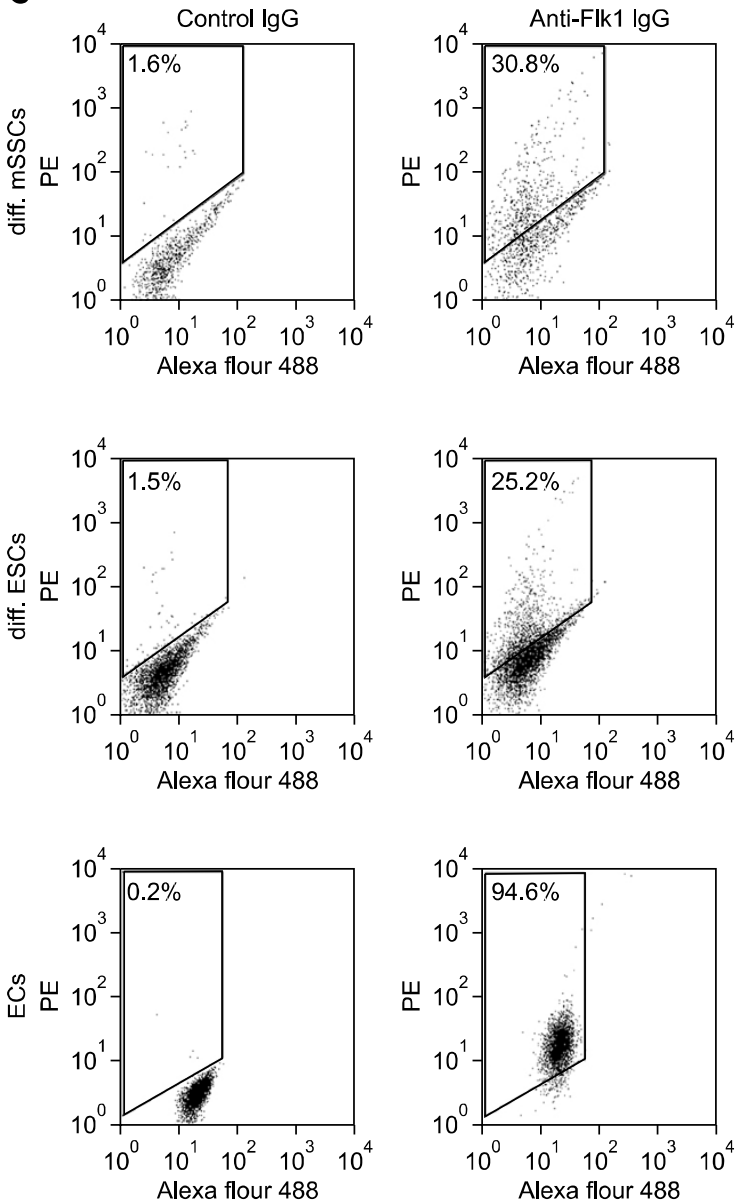
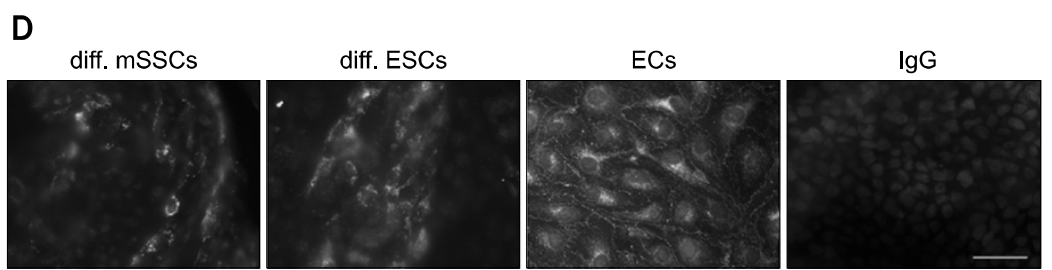

Figure 2. Mesodermal differentiation of mSSCs. (A, B) mRNA profiles of pluripotency-related marker genes (A: Oct3/4, Nanog) and mesoderm lineage specific genes (B: Brachyury, Flk1, Mesp1, Islet1, Nkx2.5) in mSSCs and ESCs during in vitro vascular differentiation process. Gene expression was measured using real-time RT-PCR and normalized as its relative ratio to GAPDH. The mRNA levels are expressed relative to the corresponding mRNA level at day 0 (undifferentiated mSSCs and ESCs; set as 1). The data were obtained from 3 independent preparations and are expressed as mean \pm SEM. (C, D) Representative FACS histograms and immunofluorescent images showing Flk1 expression in differentiated mSSCs (diff. mSSCs) and differentiated ESCs (diff. ESCs). Approximately $25 \%$ and $30 \%$ of differentiated cells from mSSCs and ESCs on day 9 of vascular differentiation were positively stained with anti-Flk1 lgG. Murine ECs were included as a positive control. Each histogram in $(C)$ is representative of three separate experiments. In addition, cells on day 14 were stained with anti-Flk1 or nonspecific control lgGs. Scale bar is $50 \mu \mathrm{m}$. Nuclei were stained with DAPI.

\section{Differentiation of mSSCs into vascular endothelial cells}

To examine whether mSSCs differentiate into vascular endothelial cells (ECs), the expression of vascular endothelial (VE)-cadherin and CD31 was analyzed using real time RT-PCR. In both mSSCs and ESCs, mRNA levels of VE-cadherin continuously in- creased as differentiation progressed until day 14 (Figure 3A). CD31 was highly expressed in undifferentiated ESCs and mSSCs, consistent with a previous report demonstrating that pluripotent cells in the inner cell mass of mouse blastocysts are strongly positive for CD31 (Robson et al., 2001). In mSSCs and ESCs, the expression of CD31 dramati- 
A

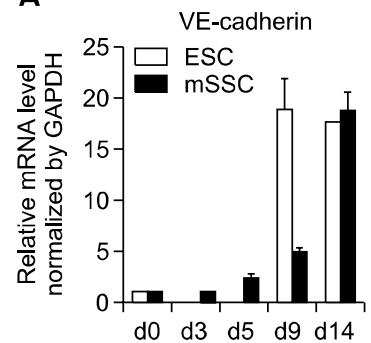

B

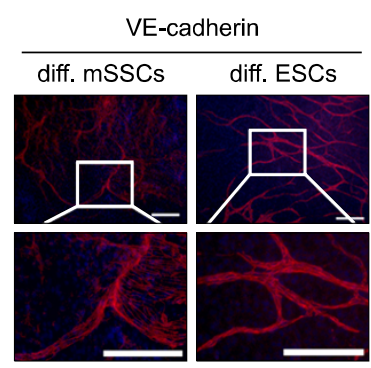

ECs

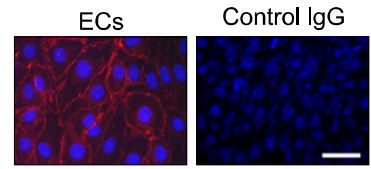

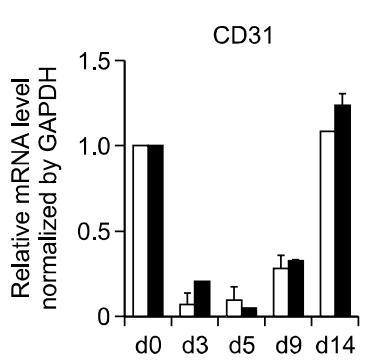

C
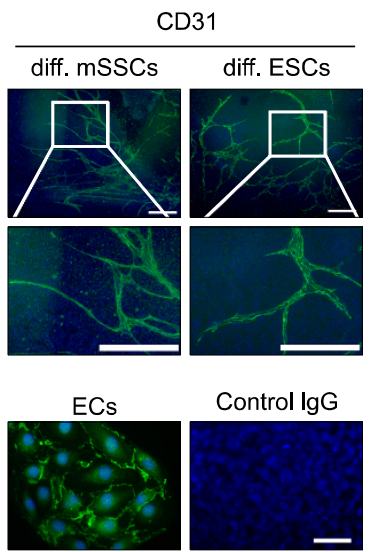

D
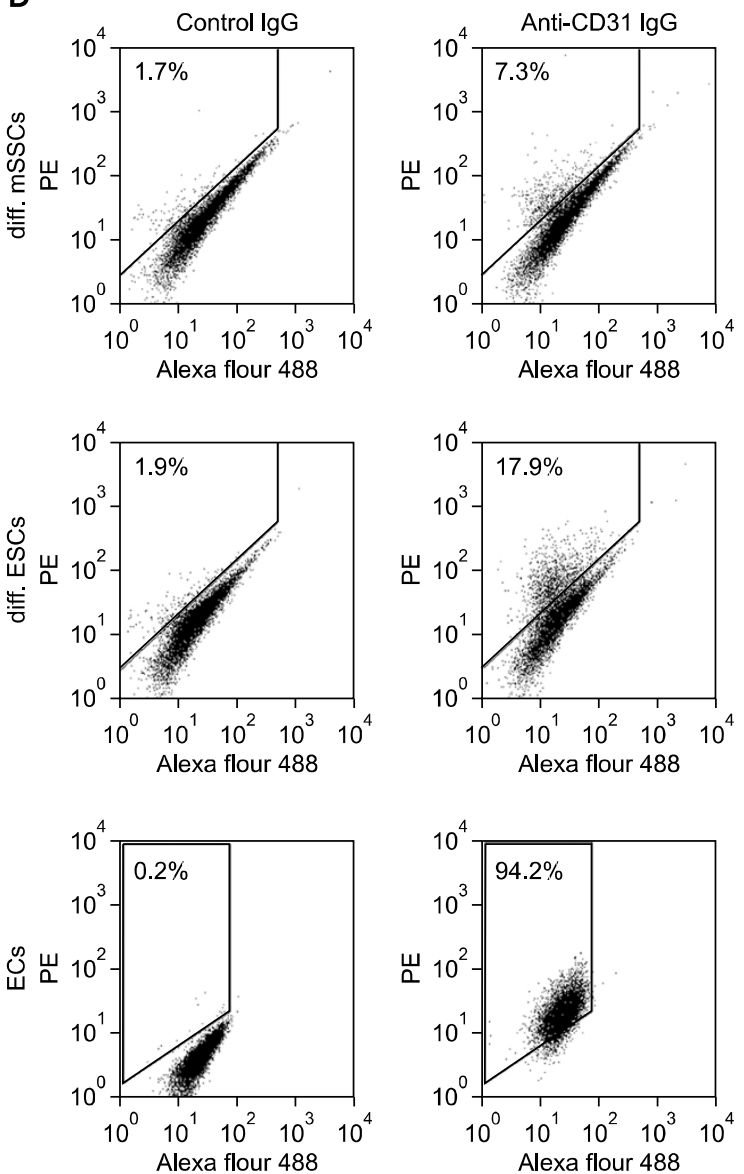

Figure 3. Vascular endothelial differentiation of mSSCs. (A) mRNA profiles of endothelial specific marker genes, VE-cadherin and CD31, in mSSCs and ESCs during in vitro vascular differentiation process. mRNA levels were measured using real-time RT-PCR and normalized as its relative ratio to GAPDH. The mRNA levels were expressed relative to the corresponding mRNA level at day 0 (undifferentiated mSSCs and ESCs; set as 1). The data were obtained from 3 independent preparations and are expressed as mean \pm SEM. (B, C) Representative images showing the expression of VE-cadherin (B) and CD31(C) proteins in differentiated mSSCs (diff. mSSCs) and differentiated ESCs (diff. ESCs). On day 14 of in vitro vascular differentiation, cells were immunostained with anti-VE-cadherin, anti-CD31 or nonspecific control lgGs. Murine ECs were included as a positive control. Scale bar is $200 \mu \mathrm{m}$. Nuclei were stained with DAPI. (D) Representative FACS histograms showing the expression of CD31 in differentiated mSSCs (diff. mSSCs) and differentiated ESCs (diff. ESCs). Approximately $7 \%$ and $18 \%$ of differentiated cells from mSSCs and ESCs on day 14 were positive for CD31. Murine ECs were included as a positive control. Each histogram in (D) is a representative of three separate experiments.

cally decreased from the beginning of differentiation until day 5 and then continued to increase until the end of the vascular differentiation process (Figure $3 A$ ). Immunocytochemical analysis also revealed that cells differentiated from mSSCs and ESCs expressed VE-cadherin or CD31 proteins at their cell-cell junctions as observed in ECs, and these VE-cadherin- or CD31-positive cells formed sprouted branch-like structures (Figures $3 \mathrm{~B}$ and $3 \mathrm{C}$ ). When the vascular endothelial differentiation efficiency was assessed using FACS analysis, approximately $7 \%$ and $18 \%$ of differentiated cells from $\mathrm{mSSC}$ and ESCs were positively stained with anti-CD31 lgG, respectively (Figure 3D).

\section{Differentiation of mSSCs into vascular smooth muscle cells}

To investigate whether mSSCs differentiate into vascular smooth muscle cells (VSMCs), expression profiles of VSMC-specific markers such as myocardin and $\alpha$-smooth muscle actin (SMA) were analyzed using real time RT-PCR. In both mSSCs and ESCs, mRNA levels of both myocardin and $\alpha$-SMA substantially increased until day 14 (Figure 4A). Immunocytochemical analysis in Figure 4B revealed that some differentiated cells from mSSCs and ESCs were co-stained with anti-SM22- $\alpha$ and anti- $\alpha$-SMA IgGs, typical markers of adult VSMCs, and exhibited the intracellular fibril structure seen in the VSMC control (Figure 4B). 
A
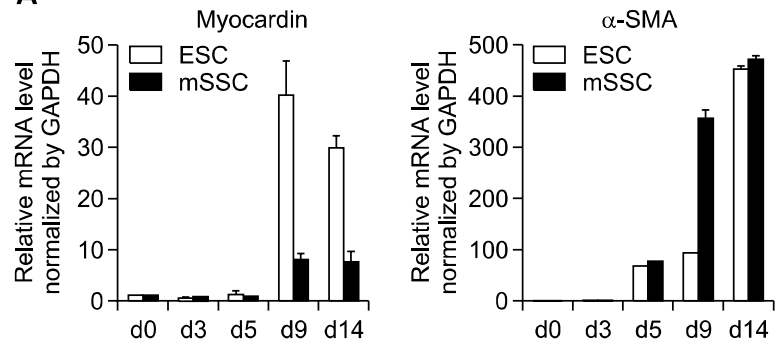

B

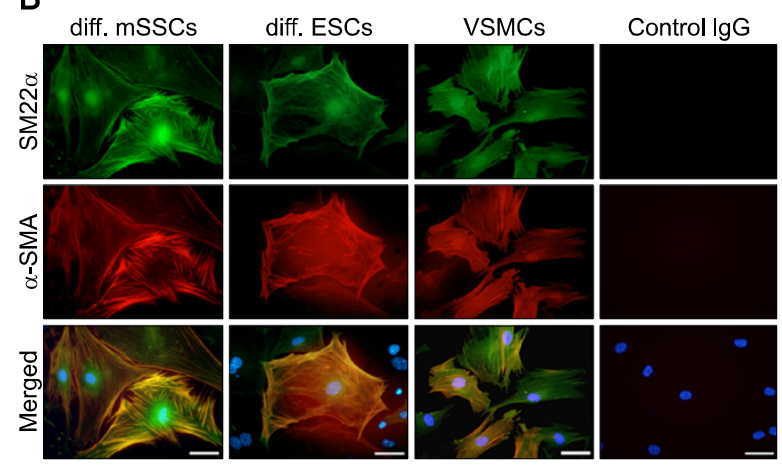

Figure 4. Vascular smooth muscle cell differentiation of mSSCs. (A) mRNA profiles of VSMC-specific genes, myocardin and $\alpha$-SMA, in $\mathrm{mSSC}$ and ESCs during in vitro vascular differentiation. The mRNA level was measured using real-time RT-PCR and normalized as its relative ratio to GAPDH. The mRNA levels were expressed relative to the corresponding mRNA level at day 0 (undifferentiated mSSCs and ESCs; set as 1). The data were obtained from 3 independent preparations and are expressed as mean \pm SEM. (B) Representative images showing the expression of SM22 $\alpha$ and $\alpha$-SMA proteins in differentiated cells from mSSCs and ESCs. On $14^{\text {th }}$ day of in vitro vascular differentiation, cells were immunostained with anti-SM22 $\alpha$ (green), anti- $\alpha$-SMA (red), or nonspecific control lgGs. VSMCs were included as a positive control. Scale bar is $50 \mu \mathrm{m}$. Nuclei were stained with DAPI.

\section{Discussion}

Stem cell therapy is a novel and promising therapeutic approach for the treatment of many incurable diseases. As such, it has become crucial in translational stem cell research to obtain autologous stem cells with ESC-like pluripotency and self renewal. To that end, many studies have attempted to dedifferentiate somatic cells into pluripotent stem cells by using somatic nuclear transfer or transcription factor-based direct reprogramming methods. However, there are many hurdles to overcome before these reprogramming methodologies can be clinically used to generate patient-specific pluripotent stem cells, such as ethical issues and safety concerns associated with the use of oocytes and genetic modification techniques. As an alternative, recent studies have identified a natural source of pluripotent stem cells. Specifically, spermatogonial stem cells which normally differentiate into sperm in the testes, can be successfully converted to multipotent stem cells with ESC-like characteristics, termed mSSCs (Kanatsu-Shinohara et al., 2004; Guan et al., 2006; Seandel et al., 2007; Conrad et al., 2008; Kossack et al., 2009). Therefore, mSSCs may be a source for autologous pluripotent stem cells without the problems associated with ESCs and induced pluripotent stem cells (de Rooij and Mizrak, 2008; Skutella, 2009).

In order to determine whether mSSCs can be used as an alternative to ESCs for the treatment of ischemic vascular diseases, the present study assessed the differentiation potential of mSSCs into VSMCs and ECs. During the in vitro differentiation process, mSSCs showed a gradual increase in the expression of early mesodermal markers, Brachyury, Flk1, and Mesp1, and a concomitant decrease in the expression of the pluripotency markers, Oct4 and Nanog. This differentiation pattern was similar to that of ESCs. However, mSSCs were slower than ESCs in the expression of transcription factors for early cardiac development, Islet1 and Nkx2.5. This may reflect a low potential of $\mathrm{mSSC}$ s to differentiate into the cardiac lineage as compared with ESCs. In addition, early cardiac transcription factor such as GATA4, ANF and Nkx2.5 expressing adult heart side population (SP) cells differentiated into von Willebrand factor (vWF)- and CD31-positive endothelial cells (Yoon et al., 2007). Further differentiation of mSSCs and ESCs into VSMCs and ECs was confirmed by the expression of cell type-specific genes. The expression levels of VE-cadherin and myocardin were slightly reduced on day 9 after induction of differentiation; the overall expression profile of vascular genes including CD31 and $\alpha$-SMA, was similar in both mSSCs and ESCs on day 14. This may indicate that mSSCs differentiate more slowly at the early vascular differentiation stages, but then accelerate to achieve similar differentiation pattern as ESCs. In addition, immunocytochemistry results showed that cells differentiated from both mSSCs and ESCs highly expressed endothelial-specific proteins, such as VE-cadherin and CD31, at cell-cell junctions and formed sprouting branch-like structures. These features mimic the embryonic vascular development process. Some differentiated cells from mSSCs and ESCs expressed both $\alpha$-SMA and SM22 $\alpha$, and exhibited the intracellular fibril structure characteristic of VSMC.

In summary, we demonstrated that mSSCs, like ESCs can be efficiently differentiated into VSMCs and ECs in vitro. The vascular differentiation process of mSSC displayed a developmentally appropriate sequence of transcription factor expression, similar to ESCs, indicating that mSSCs and ESCs have similar self-renewal and differentiation 
properties. Our findings suggest that mSSCs are as suitable as ESCs as a source of VSMCs and ECs for basic research and potentially therapeutic applications.

\section{Methods}

\section{Cell culture}

Established mSSCs (CHA_mSSC-1) and murine ESCs (D3, ATCC, Manassas, VA) were cultured on mitomycin-C (Sigma, Louis, MO) - treated mouse embryonic fibroblasts (STO, ATCC) in Dulbecco's modified Eagle's medium (DMEM, Gibco, Carlsbad, CA) supplemented with $20 \%$ serum replacement (SR, Gibco), 2-mercaptoethanol (50 $\mu \mathrm{M}$, Gibco), nonessential amino acids (Gibco), penicillin (50 $\mathrm{U} / \mathrm{ml}$, Gibco), streptomycin (50 U/ml, Gibco), and human recombinant leukemia inhibitory factor $\left(10^{3} \mathrm{U} / \mathrm{ml}\right.$, Millipore, Temecular, CA) (Kim et al., 2010). The murine ECs (ATCC) and STO were cultured in DMEM containing $10 \%$ of fetal bovine serum (FBS; Gibco). VSMCs (ScienCell, Carsbad, $\mathrm{CA}$ ) were cultivated in smooth muscle cell culture medium (ScienCell).

\section{In vitro vascular differentiation}

Prior to the in vitro differentiation, mSSCs and ESCs were cultured on gelatin (Sigma)-coated dishes without feeder cells for 2 days. They were then aggregated into EBs using standard handing drop method. In brief, mSSCs or ESCs $\left(10^{3}\right.$ cells $\left./ \mathrm{ml}\right)$ were suspended in DMEM/F12 medium containing BMP4 (10 ng/ml; Prospecbio, East Brunswick, NJ), VEGF (10 ng/ml;, R\&D systems Inc., Minneapolis MN), activin A ( $3 \mathrm{ng} / \mathrm{ml}$, Prospecbio), bFGF ( $5 \mathrm{ng} / \mathrm{ml}$; Prospecbio), $10 \%$ of SR, and 2-mercaptoethanol. $20 \mu \mathrm{l}$ of cell suspension was placed on the lids of petri dishes and cultured in hanging drops for 2 days. Floating EBs were further cultured in bacteriological petri dishes filled with fresh differentiation medium for another 2 days. EBs were then transferred to gelatin-coated cell culture dishes filled with basal medium supplemented with $2 \%$ of FBS, VEGF, bFGF, epidermal growth factor, and insulin growth factor (Lonza, Basel, Switzerland) unitl day $14^{\text {th }}$.

\section{RT-PCR analysis}

Total RNA was extracted from cells using Trizol (Invitrogen, Carlsbad, CA) and cDNA was synthesized using the Superscript first-strand synthesis system (Invitrogen). Real-time PCR was performed with a SYBR green PCR kit (Qiagen, Valencia, CA) using a real-time PCR systems (Applied Biosystems, Forster city, CA). Specific primer sequences are listed in Supplemental Data Table S1. Data were analyzed using the $\Delta \Delta \mathrm{Ct}$ method. The raw data were normalized to those of the housekeeping gene, glyceraldehyde- 3-phosphate dehydrogenase (GAPDH). All reactions were performed in triplicate.

\section{Immunocytochemistry}

EBs attached to chamber slides were fixed in $4 \%$ paraf- ormaldehyde (Sigma), permeabilized with $0.2 \%$ Triton $\mathrm{X}-100$ (Sigma), and blocked in $10 \%$ normal goat serum (Vector Laboratories Inc., Burlingame, CA). Next, EBs were incubated with primary IgGs against Flk1 (Cell signaling technology Inc. Danvers, MA), VE-cadherin (BD Pharmingen, Bedford, MA), CD31 (BD Pharmingen), SM22 $\alpha$ (Abcam, Cambridge, MA), $\alpha$-SMA (Sigma-Aldrich, MO), or nonspecific control IgGs. Then, EBs were incubated with fluorescence-conjugated secondary IgGs (Chemicon, Temecular, CA) and mounted with VectaShield mounting medium containing 4, 6-diamidino-2-phenylindole (DAPI). Immunofluorescence was analyzed using a fluorescence microscope (Carl Zeiss, Oberkochen, Germany).

\section{FACS analysis}

Cell surface expression of Flk1 and CD31 was analyzed by FACS using a FACSAaria flow cytometer (BD Biosciences). Cells pretreated with human-FcR blocking reagent (Miltenyi Biotec, Bergisch, Galdbach, Germany) were labeled with PE-conjugated anti-Flk1 (BD Pharmigen) or anti-CD31 (BD Pharmigen) IgGs for $2 \mathrm{~h}$ at $4^{\circ} \mathrm{C}$, and then washed with ice-cold buffer. Samples were analyzed along with negative controls stained with appropriate isotype-matched nonspecific lgGs.

\section{Statistical analysis}

All data were presented as mean \pm standard error of the mean (SEM).

\section{Supplemental data}

Supplemental data include two tables and four figures and can be found with this article online at http://e-emm.or.kr/ article/article_files/SP-44-4-06.pdf.

\section{Acknowledgements}

This work was supported by a grant of the Korea Healthcare Technology R \& D Project, Ministry of Health, Welfare \& Family Affairs, Republic of Korea (A100638).

\section{References}

Asahara T, Murohara T, Sullivan A, Silver M, van der Zee R, Li T, Witzenbichler B, Schatteman G, Isner JM. Isolation of putative progenitor endothelial cells for angiogenesis. Science 1997;275:964-7

Conrad S, Renninger M, Hennenlotter J, Wiesner T, Just L, Bonin M, Aicher W, Buhring HJ, Mattheus U, Mack A, Wagner HJ, Minger S, Matzkies M, Reppel M, Hescheler J, Sievert KD, Stenzl A, Skutella T. Generation of pluripotent stem cells from adult human testis. Nature 2008;456:344-9

de Rooij DG, Mizrak SC. Deriving multipotent stem cells from mouse spermatogonial stem cells: a new tool for developmental and clinical research. Development 2008; 135:2207-13 
Guan K, Nayernia K, Maier LS, Wagner S, Dressel R, Lee JH, Nolte J, Wolf F, Li M, Engel W, Hasenfuss G. Pluripotency of spermatogonial stem cells from adult mouse testis. Nature 2006;440:1199-203

Hill JM, Zalos G, Halcox JP, Schenke WH, Waclawiw MA, Quyyumi AA, Finkel T. Circulating endothelial progenitor cells, vascular function, and cardiovascular risk. $\mathrm{N}$ Engl J Med 2003;348:593-600

Kanatsu-Shinohara M, Inoue K, Lee J, Yoshimoto M, Ogonuki N, Miki H, Baba S, Kato T, Kazuki Y, Toyokuni S, Toyoshima M, Niwa O, Oshimura M, Heike T, Nakahata T, Ishino F, Ogura A, Shinohara T. Generation of pluripotent stem cells from neonatal mouse testis. Cell 2004;119: 1001-12

Kim HJ, Lee HJ, Lim JJ, Kwak KH, Kim JS, Kim JH, Han YM, Kim KS, Lee DR. Identification of an intermediate state as spermatogonial stem cells reprogram to multipotent cells. Mol Cells 2010;29:519-26

Kossack N, Meneses J, Shefi S, Nguyen HN, Chavez S, Nicholas C, Gromoll J, Turek PJ, Reijo-Pera RA. Isolation and characterization of pluripotent human spermatogonial stem cell-derived cells. Stem Cells 2009;27:138-49

Park JH, Yoon JY, Ko SM, Jin SA, Kim JH, Cho CH, Kim JM, Lee JH, Choi SW, Seong IW, Jeong JO. Endothelial progenitor cell transplantation decreases lymphangiogenesis and adverse myocardial remodeling in a mouse model of acute myocardial infarction. Exp Mol Med 2011;43:479-85
Pearson S, Sroczynska P, Lacaud G, Kouskoff V. The stepwise specification of embryonic stem cells to hematopoietic fate is driven by sequential exposure to Bmp4, activin A, bFGF and VEGF. Development 2008;135:1525-35

Rafii S, Lyden D. Therapeutic stem and progenitor cell transplantation for organ vascularization and regeneration. Nat Med 2003;9:702-12

Robson P, Stein P, Zhou B, Schultz RM, Baldwin HS. Inner cell mass-specific expression of a cell adhesion molecule (PECAM-1/CD31) in the mouse blastocyst. Dev Biol 2001; 234:317-29

Seandel M, James D, Shmelkov SV, Falciatori I, Kim J, Chavala S, Scherr DS, Zhang F, Torres R, Gale NW, Yancopoulos GD, Murphy A, Valenzuela DM, Hobbs RM, Pandolfi PP, Rafii S. Generation of functional multipotent adult stem cells from GPR125+ germline progenitors. Nature 2007; 449:346-50

Shantsila E, Watson T, Lip GY. Endothelial progenitor cells in cardiovascular disorders. J Am Coll Cardiol 2007;49: 741-52

Skutella T. Induced pluripotent stem cells from adult testis: a new source of stem cells? Regen Med 2009;4:3-5

Yoon J, Choi SC, Park CY, Shim WJ, Lim DS. Cardiac side population cells exhibit endothelial differentiation potential. Exp Mol Med 2007;39:653-62 